BMJ Open

Diabetes

Research

\& Care

\title{
Plasma glucose and insulin response to two oral nutrition supplements in adults with type 2 diabetes mellitus
}

\author{
Maureen B Huhmann, ${ }^{1}$ Kristen N Smith, ${ }^{1}$ Sherwyn L Schwartz, ${ }^{2}$ Stacie K Haller, ${ }^{2}$ \\ Sarah Irvin, ${ }^{3}$ Sarah S Cohen ${ }^{3}$
}

To cite: Huhmann MB Smith KN, Schwartz SL, et al. Plasma glucose and insulin response to two oral nutrition supplements in adults with type 2 diabetes mellitus. BMJ Open Diabetes Research and Care 2016:4:e000240. doi:10.1136/bmjdrc-2016000240

Received 8 April 2016 Revised 2 August 2016 Accepted 11 August 2016

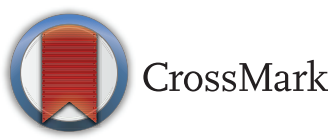

\footnotetext{
${ }^{1}$ Department of Clinical Sciences, Nestle Health Science, Florham Park, New Jersey, USA

${ }^{2}$ Diabetes \& Glandular Disease Clinic, San Antonio, Texas, USA

${ }^{3}$ EpidStat Institute, Ann Arbor, Michigan, USA
}

Correspondence to Dr Maureen B Huhmann; Maureen.huhmann@us. nestle.com

\section{ABSTRACT}

Objective: The purpose of this clinical trial was to compare the glucose usage of two oral nutritional supplement (ONS) products and to assess whether a diabetes-specific formulation provides improved glucose stabilization and management compared with a standard formula.

Research design and methods: A total of 12 subjects with type 2 diabetes ( 7 males and 5 females) completed a randomized, cross-over design trial. Each subject consumed isocaloric amounts of either the standard ONS or the diabetes-specific formula ONS on different dates, 1 week apart. Glucose and insulin measures were recorded at baseline, and 10, 20,30, $60,90,120,150,180,210$ and 240 min after the beverage was consumed and then used to calculate area under the curve (AUC) for each subject.

Results: The mean glucose AUC was lower in the diabetes-specific ONS group than in the standard group $(p<0.0001)$, but there was not a significant difference observed for mean insulin AUC $(p=0.068)$. A sensitivity analysis of the mean insulin AUC measures was performed by removing a potential outlier from the analysis, and this resulted in a significant difference between the groups $(p=0.012)$. First-phase insulin measures and an insulinogenic index calculated for the beverages showed no significant differences.

Conclusions: On the basis of the results of this trial of 12 subjects, the diabetes-specific ONS appears to provide better glucose maintenance in persons with type 2 diabetes when compared to the standard formula ONS.

Trial registration number: NCT02612675.

\section{INTRODUCTION}

Diabetes is a common disease affecting about $6 \%$ of the population in the USA, with another 1.3 million diagnosed each year. ${ }^{1}$ Type 2 diabetes is common in older Americans and nearly $18 \%$ of Americans aged $\geq 60$ years are afflicted with this disease. ${ }^{1}$ These statistics suggest that the prevalence of diabetes will continue to increase as the number and proportion of older Americans also increases.

When diabetes is not well controlled, harmful physiological consequences such as

\section{Key messages}

- Diabetes-specific oral nutritional supplement (ONS) has been developed to assist people with diabetes in meeting nutrient needs.

- Consumption of a diabetes-specific ONS leads to a lower mean glucose response than a standard ONS.

- Consumption of a diabetes-specific ONS leads to lower insulin response when the analysis controlled for an outlier.

dehydration, poor wound healing, chronic infections, fatigue, blurred vision, electrolyte imbalances and weight loss may occur. The prevention of these direct diabetes-related outcomes can have a profound effect on the quality and duration of the lives of individuals living with diabetes. Indeed, the Diabetes Control and Complications Trial (DCCT) identified that maintenance of near normal blood glucose levels in diabetic individuals over 10 years reduced the risk for eye disease by $76 \%$, kidney disease by $35-54 \%$, and nerve deterioration by $60 \%$. This trial also identified a direct correlation between the level of glucose control and development of diabetes-related complications. ${ }^{2}$ Thus, adequate diabetes control is critical for the prevention of short-term and long-term complications.

Management of type 2 diabetes requires a balanced diet coupled with monitoring of blood glucose and, in some cases, medication. ${ }^{3}{ }^{4}$ Medical nutrition therapy is an essential component of metabolic control for patients with type 2 diabetes mellitus. ${ }^{5}$ The current recommendations of the American Diabetes Association emphasize an individualized approach to nutritional care, but suggest that $15-20 \%$ of the daily calories is from protein and $<10 \%$ is from saturated fat. The remainder of the calories is from carbohydrate and monounsaturated and polyunsaturated fats. Fiber is recommended 
for good bowel health; adequate amounts have shown benefits on glycemic control, hyperinsulinemia, and plasma lipids. ${ }^{35}$

Oral nutritional supplements (ONS) are often used to supplement the diet with shortfall nutrients such as protein, vitamins, and minerals. ${ }^{6}$ In some instances, they may be used as an occasional meal replacement. There are many oral nutritional supplement (ONS) available for clinicians to choose from. An important factor in the decision on the appropriateness of an ONS for a specific patient is the potential effect of the ONS on their medical condition. A systematic review by Elia et $a l^{7}$ showed that the incorporation of diabetes-specific formulas is associated with improvements in glycemic control, compared with standard formulations. The purpose of this study was to experimentally compare the effect of a single administration of two liquid ONS (a standard formula compared with a diabetes-specific formula designed for individuals with impaired glucose tolerance) on glucose and insulin metabolism in subjects with type 2 diabetes.

\section{RESEARCH DESIGN AND METHODS \\ Study design and clinical protocols}

This study was a randomized, crossover clinical trial of 12 subjects with type 2 diabetes, randomized to one of the two separate interventions on two separate study days, 1 week apart. The protocol design was based on the FDA Draft Guidance on Bioavailability and Bioequivalence Studies. Study recruitment and data collection took place in January 2014 at Diabetes and Glandular Disease Clinic in San Antonio, Texas, USA. Subjects were between 20 and 75 years of age with type 2 diabetes controlled with diet or diet plus oral agents, with the exception of sulfonylureas such as glimepiride (Amaryl), glipizide (Glucotrol/Glucotrol XL) and glyburide (DiaBeta, Micronase, Glynase Prestabs), meglinitides such as reaglinide (Prandin) and nateglinide (Starlix) and $\alpha$-glucosidase inhibitors such as acarbose (Precose) and miglitol (Glyset). Diabetic control was defined as having hemoglobin A1C levels $<9.0 \%$ ( $75 \mathrm{mmol} / \mathrm{mol}$ ) and fasting blood glucose levels $<180 \mathrm{mg} / \mathrm{dL}$. Subjects were excluded from this study if they met any of the following measures: abnormal thyroid function, creatinine levels $>2.0 \mathrm{mg} / \mathrm{dL}$, potassium $<3.5 \mathrm{mEq} / \mathrm{L}$, gastrointestinal disease (including ulcer, gastritis, diarrhea, gastroparesis, and vomiting), current unstable diabetes, or undergoing treatment for cancer, heart disease, or renal disease. Additional exclusion criteria involved the use of current insulin therapy or insulin therapy within the previous month, pregnancy, or subject's inability to give informed consent or follow directions. Allergies to milk, soy, or any component of the test product were also cause for exclusion.

The study was approved by the ethics committee of the participating facility and fulfilled all requirements for human research, including Declaration of Helsinki and Good Clinical Practice; all subjects provided written informed consent prior to the start of the study.

Subjects were recruited from the clinic waiting room using flyers and by practitioners at the facility. Qualified subjects were randomly assigned to one of the two intervention groups and then were crossed over to the alternate group. The interventions were provided 1 week apart. Study products were chilled and poured into open cups by the facility. Subjects ingested one of the two treatments at each test visit, with the visit order selected randomly.

\section{Clinic visits}

Subjects had a screening visit and two subsequent visits separated by a washout period of 7 days. Informed consent and medical history were obtained at screening as well as samples for serum chemistry (including HbAlc, creatinine, potassium, TSH, and Free T4) and a brief physical examination. Weight and vital signs were measured at all clinic visits. After an overnight fast of at least 8 hours, participants received an intravenous line for blood withdrawal. Subjects that were taking Metformin were instructed to hold their medication the morning of the testing and to resume their medication following the 4-hour testing period. At time 0, a blood sample for fasting glucose and insulin levels was drawn. Subjects were instructed not to consume oral diabetes medications before or with the randomly assigned intervention or during the 4-hour intervention visit. Subjects then consumed the randomly assigned intervention product (within $10 \mathrm{~min}$ ). They were also instructed to remain recumbent, to refrain from smoking, and not to consume any additional food or beverage during the course of the visit. Blood samples for glucose and insulin levels were drawn at 10, 20, 30, 60, 90, 120, 150, 180, 210 and $240 \mathrm{~min}$ after the beverage was consumed.

\section{Test products}

The test products, beverage A (diabetes-specific, Boost Glucose Control) and beverage B (standard, Boost Original), were provided in isocaloric amounts. Both test products provided 190 calories; diabetes-specific ONS provided $16 \mathrm{~g}$ protein, $16 \mathrm{~g}$ carbohydrate, and $7 \mathrm{~g}$ fat while standard ONS provided $8 \mathrm{~g}$ protein, $32 \mathrm{~g}$ carbohydrate, and $3 \mathrm{~g}$ fat. A full description of the nutritional profile of both beverages is shown in table 1 .

\section{Laboratory measurements}

At each study visit, blood samples from each subject were obtained via an indwelling catheter and drawn by one of the research team staff in the morning. A total of $80 \mathrm{~mL}$ of serum/plasma was drawn at each test visit. Glucose was analyzed using the hexokinase G-6-PDH method. Insulin was analyzed using ST AIA-PACK IRI. All parameters were analyzed by Diabetes \& Glandular Disease Clinic, Pennsylvania, USA. 


\section{Sample size}

In a previous study with 10 subjects who received two different enteral feeding products, the SD of the difference of areas under the curve was $74 \mathrm{mg} / \mathrm{dL} / 4$ hours. ${ }^{8}$ Using these numbers, sample sizes were calculated using an $\alpha$ of 0.05 and a $\beta$ of 0.2 . To detect a difference of $90 \mathrm{mg} / \mathrm{dL}$, a sample size of 11 was required. Therefore, for this study, a sample size of 12 was used to ensure adequate statistical power.

\section{Statistical analysis}

All data were collected on paper case report forms, which were entered into an Excel database and checked by two separate investigators for correctness and completeness. Statistical analyses were performed using Excel. Area under the curve (AUC) values was calculated for each of the 12 patients. Four AUC calculations were performed per patient: diabetes-specific ONS-AUC for glucose, diabetes-specific ONS-AUC for insulin, standard ONSAUC for glucose, standard ONS-AUC for insulin.

The AUC calculation was performed using the trapezoid rule, and integrals for each time interval and change from baseline reading were recorded up to the $240 \mathrm{~min}$ end time. The calculus integral formula was adapted for

\section{Table 1 Nutritional profile of test beverages}

\begin{tabular}{|c|c|c|}
\hline & $\begin{array}{l}\text { Diabetes-specific } \\
\text { ONS: } 240 \mathrm{~mL}\end{array}$ & $\begin{array}{l}\text { Standard } \\
\text { ONS: } \\
190 \mathrm{~mL}\end{array}$ \\
\hline $\begin{array}{l}\text { Caloric density (kcal/ } \\
\text { study serving) }\end{array}$ & 190 & 190 \\
\hline Protein (g) & 16 & 8 \\
\hline Fat $(\mathrm{g})$ & 7 & 3 \\
\hline Carbohydrates (g) & 16 & 32 \\
\hline $\begin{array}{l}\text { Fiber } \\
\text { (fructooligosaccharides } \\
\text { and inulin) (g) }\end{array}$ & 3 & 0 \\
\hline $\mathrm{Na}(\mathrm{mg})$ & 220 & 119 \\
\hline $\mathrm{K}(\mathrm{mg})$ & 50 & 364 \\
\hline $\mathrm{Cl}(\mathrm{mg})$ & 136 & 215 \\
\hline $\mathrm{Ca}(\mathrm{mg})$ & 350 & 238 \\
\hline$P(\mathrm{mg})$ & 300 & 238 \\
\hline $\mathrm{Ca} / \mathrm{P}$ (calculated) & 1.17 & 0 \\
\hline $\mathrm{Mg}(\mathrm{mg})$ & 40 & 79 \\
\hline$M n(\mu g)$ & 0.7 & 0.6 \\
\hline$A(I U)$ & 500 & 990 \\
\hline$\beta$ Carotene & 250 & 495 \\
\hline D (IU) & 100 & 190 \\
\hline$E(I U)$ & 60 & 24 \\
\hline $\mathrm{K} 1(\mu \mathrm{g})$ & 32 & 48 \\
\hline $\mathrm{C}(\mathrm{mg})$ & 102 & 48 \\
\hline $\mathrm{B} 1$ (mg) & 0.38 & 0.30 \\
\hline $\mathrm{B} 2$ (mg) & 0.43 & 0.34 \\
\hline Niacin (mg) & 5 & 3 \\
\hline B6 (mg) & 1 & 0.6 \\
\hline Folic acid $(\mu \mathrm{g})$ & 200 & 79 \\
\hline Pantothenic acid (mg) & 2.5 & 2 \\
\hline $\mathrm{B} 12(\mu \mathrm{g})$ & 3 & 1.7 \\
\hline Biotin $(\mu \mathrm{g})$ & 75 & 59 \\
\hline
\end{tabular}

Excel and used to calculate each integral. After AUCs were calculated for each measure, means of the AUCs were calculated for each group, and paired t-tests were used to assess the potential difference between the means of each formula group. A $p$ value of $<0.05$ was used to determine the statistical significance. All $\mathrm{p}$ values reported are two-sided. Additionally, an insulinogenic index was calculated for each patient as follows: the difference between insulin measures taken at baseline and at 30 min was divided by the difference between glucose levels at baseline and $30 \mathrm{~min}$. This value was multiplied by a conversion factor of 0.0555 to obtain the insulinogenic indices for each group, or the measure of insulin secretion at $30 \mathrm{~min}$ for each patient in $\mathrm{mmol} / \mathrm{L}$.

\section{RESULTS}

Thirteen subjects were screened and randomized into the trial; however, one subject was unable to complete the study visits. Analytics were conducted for the 12 subjects with complete data. Of the subjects, $41.7 \%$ (5 of 12) were female and $58.3 \%$ (7 of 12) were male. Subjects had a mean age of 59.5 years (table 2 ).

\section{Glucose and insulin responses}

No significant differences between fasting glucose or insulin were identified (glucose $\mathrm{p}=0.85$; insulin $\mathrm{p}=0.85$ ).

Table 2 Demographic and baseline characteristics of subjects

\begin{tabular}{ll}
\hline Characteristic & Value \\
\hline Sex & \\
$\quad$ Male & $7(58 \%)$ \\
Female & $5(42 \%)$ \\
Age & $59.5 \pm 9.59$ \\
Height (cm) & 171.67 \\
& \pm 10.58 \\
Weight (kg) & $91.48 \pm 16.24$ \\
Body mass index $\left(\mathrm{kg} / \mathrm{m}^{2}\right)$ & $31.05 \pm 5.11$ \\
BP systolic (mm Hg) & $121 \pm 7.65$ \\
BP diastolic (mm Hg) & $76 \pm 9.95$ \\
Heart rate (bpm) & $76.17 \pm 11.86$ \\
Comorbidities & \\
$\quad$ Diabetes, type 2 & $12(100 \%)$ \\
Hypertension & $7(58 \%)$ \\
Hyperlipidemia & $6(50 \%)$ \\
Fatty liver disease & $2(17 \%)$ \\
Medication usage & \\
$\quad$ Metformin & $10(83 \%)$ \\
Fish oil & $1(8 \%)$ \\
Antihyperlipidemic drugs & $6(50 \%)$ \\
Antihypertensive drugs & $7(58 \%)$ \\
Dietary supplements (ie, vitamins, & $7(58 \%)$ \\
minerals, herbs) & \\
Other drugs (ie, diuretic, antihistamine, H2 & $10(83 \%)$ \\
receptor antagonist) & \\
\hline Data are (\%) or means 50 &
\end{tabular}

Data are $n(\%)$ or means \pm SD.

$\mathrm{BP}$, blood pressure. 
Figure 1 Mean glucose change

from baseline by treatment

product. $₫$, standard ONS;

diabetes-specific ONS.
Mean Glucose Change from Baseline Levels by Time of Sampling and Product

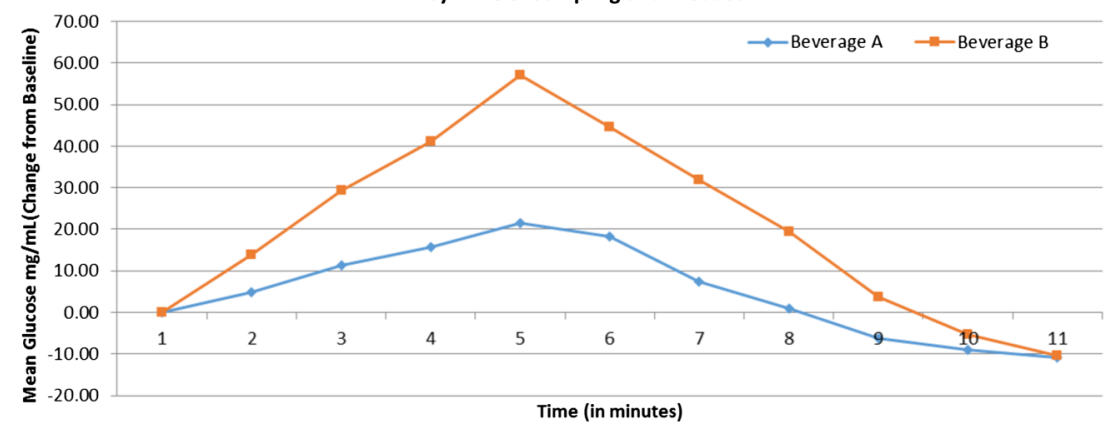

Figure 1 shows glucose response by test product. The mean glucose AUC was significantly lower in the diabetes-specific ONS group compared with standard ONS $(\mathrm{p}<0.0001)$.

Figure 2 shows insulin response by test product. There was not a significant difference observed for mean insulin AUC $(p=0.068)$. A sensitivity analysis of the mean insulin AUC measures was performed to identify any potential outliers. One outlier was identified, which was observed to have an insulin AUC value more than twice as high as the next highest value. Thus, this value was removed from analyses, resulting in a significant difference between the groups $(\mathrm{p}=0.012)$.

There were no significant differences in the first-phase insulin measure (ie, AUC calculated at the 30 min time interval) between the formula groups $(p=0.24)$.

The insulinogenic index had a high degree of variability with a mean (SD) of 103.4 (148.4) for diabetesspecific ONS and 21.2 (24.3) for standard ONS $(\mathrm{p}=0.07)$.

\section{DISCUSSION}

This study included 12 individuals with type 2 diabetes controlled either with diet or with Metformin. The results of this pilot trial demonstrate that the use of this diabetes-specific ONS designed to assist with glucose control was effective in providing better glucose maintenance for individuals with type 2 diabetes compared with a standard formula.
It is becoming increasingly accepted that people with diabetes mellitus require nutritional support to meet micronutrient and macronutrient needs in a way that will not harm glycemic control. Products specifically designed for the unique needs of persons with type 2 diabetes have been developed and marketed in recent years. These have been available as snacks or sometimes as a meal substitute. These specialized formulations have demonstrated greater benefit in the normalization of fasting and postprandial glucose concentrations. This study confirms that a product designed specifically for individuals with diabetes can assist in attaining normal blood sugars. Additionally, there may be benefits to insulin sensitivity, and with long-term use, this may reduce complications associated with diabetes, primarily cardiovascular outcomes. ${ }^{7}$ 9-11 This study provides new data which illustrates that the product tested here does in fact provide a benefit in terms of the short-term maintenance of glycemic control in individuals with type 2 diabetes.

When assessing the ability to extrapolate these results more broadly, one must look at relevant characteristics of the study population in relation to the general population. The majority of these patients suffered from additional comorbidities, including hypertension, hyperlipidemia, and fatty liver disease. A systematic review of the prevalence of hypertension in diabetics reported that $50-75 \%$ of diabetics experience hypertension. ${ }^{12}$ We observed a similar incidence in our study population. The CDC reports the incidence of
Figure 2 Mean insulin change from baseline by treatment product. $\sqsubset$, Standard ONS; diabetes-specific ONS.

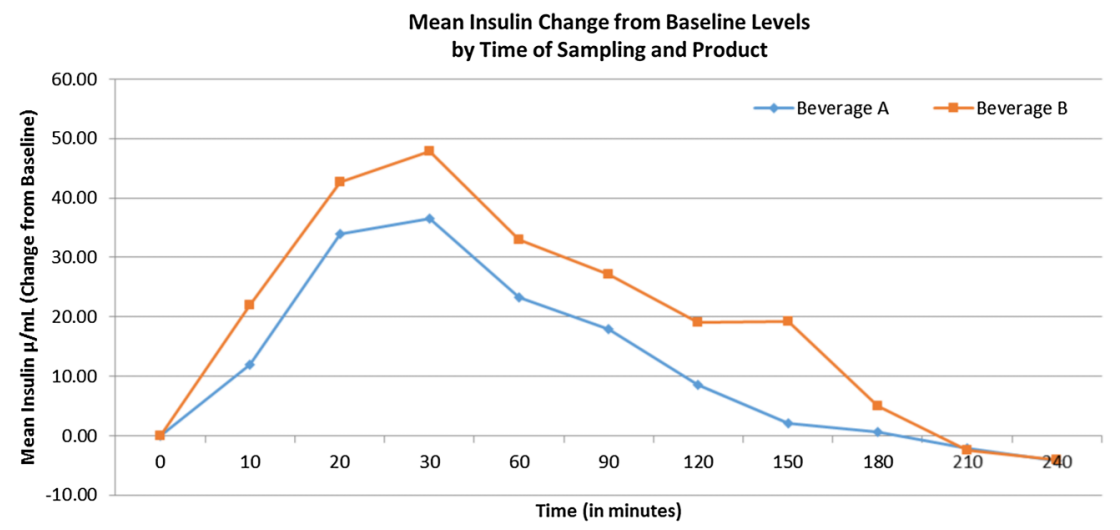


dyslipidemia in diabetics to be as high as $65 \% .{ }^{13}$ Our population and a documented incidence of $50 \%$. A recent publication found that up to $50 \%$ of patients with type 2 diabetes have non-alcoholic fatty liver disease (NAFLD). ${ }^{14}$ Only $17 \%$ of the population in this study had documented NAFLD. It is possible that the prevalence of NAFLD was higher, but the participants had not received an official diagnosis.

A strength of this trial was the use of individuals with type 2 diabetes as the study population, and the results of this trial may be extrapolated to other people with type 2 diabetes controlled with diet or Metformin as well. However, this was an acute intervention with a relatively small number of participants at only one medical facility which are limitations of this study. Additional studies are needed to further investigate the long-term effects of diabetes-specific products within a diabetes population.

\section{CONCLUSIONS}

In summary, consumption of a diabetes-specific nutritional supplement led to a significantly lower mean glucose AUC compared with subjects consuming a standard formula. These findings suggest that the use of a product designed for use by individuals with type 2 diabetes may prove useful as an adjunct to glucose stabilization and management practices.

Contributors MBH wrote protocol, reviewed data, reviewed/edited manuscript, and contributed to discussion. KNS wrote the manuscript and reviewed data. SLS researched data and reviewed the manuscript. SKH researched data and reviewed the manuscript. SI performed statistical analysis and reviewed/edited the manuscript. SSC performed statistical analysis and reviewed/edited the manuscript.

Funding Funding for this trial was provided by Nestlé Health Science.

Competing interests MBH and KNS received salary from Nestle Health Science.

Patient consent Obtained.

Ethics approval RCRC Independent Review Board, LLC.

Provenance and peer review Not commissioned; externally peer reviewed.

Data sharing statement All data collected as part of this study is presented here.
Open Access This is an Open Access article distributed in accordance with the Creative Commons Attribution Non Commercial (CC BY-NC 4.0) license, which permits others to distribute, remix, adapt, build upon this work noncommercially, and license their derivative works on different terms, provided the original work is properly cited and the use is non-commercial. See: http:// creativecommons.org/licenses/by-nc/4.0/

\section{REFERENCES}

1. Centers for Disease Control and Prevention. National Diabetes Statistics Report: Estimates of Diabetes and Its Burden in the United States, 2014. Atlanta, GA: US. Department of Health and Human Services, 2014.

2. The effect of intensive treatment of diabetes on the development and progression of long-term complications in insulin-dependent diabetes mellitus. The Diabetes Control and Complications Trial Research Group. N Engl J Med 1993, 329:977-86.

3. Franz MJ, Bantle JP, Beebe CA, et al. Nutrition principles and recommendations in diabetes. Diabetes Care 2004;27(Suppl 1): S36-46.

4. American Diabetes Association. 4. Foundations of care: education, nutrition, physical activity, smoking cessation, psychosocial care, and immunization. Diabetes Care 2015;38(Suppl):S20-30.

5. Evert AB, Boucher JL, Cypress M, et al. Nutrition therapy recommendations for the management of adults with diabetes. Diabetes Care 2013;36:3821-42.

6. Stratton RC, Elia M. A review of reviews: a new look at the evidence for oral nutritional supplements in clinical practice. Clin Nutr Suppl 2007;2:5-23.

7. Elia $\mathrm{M}$, Ceriello $\mathrm{A}$, Laube $\mathrm{H}$, et al. Enteral nutritional support and use of diabetes-specific formulas for patients with diabetes: a systematic review and meta-analysis. Diabetes Care 2005;28:2267-79.

8. Gannon MC, Nuttall FQ. Factors affecting interpretation of postprandial glucose and insulin areas. Diabetes Care 1987;10:759-63.

9. Yokoyama J, Someya Y, Yoshihara R, et al. Effects of high-monounsaturated fatty acid enteral formula versus high-carbohydrate enteral formula on plasma glucose concentration and insulin secretion in healthy individuals and diabetic patients. $J$ Int Med Res 2008;36:137-46.

10. Ceriello A, Lansink $\mathrm{M}$, Rouws $\mathrm{CH}$, et al. Administration of a new diabetes-specific enteral formula results in an improved $24 \mathrm{~h}$ glucose profile in type 2 diabetic patients. Diabetes Res Clin Pract 2009;84:259-66.

11. Li YX, Zeng JB, Yu K, et al. Beneficial effects of a diabetes specific formula on insulin sensitivity and free fatty acid in patients with type 2 diabetes mellitus. Chin Med J 2008;121:691-5.

12. Colosia AD, Palencia R, Khan S. Prevalence of hypertension and obesity in patients with type 2 diabetes mellitus in observational studies: a systematic literature review. Diabetes Metab Syndr Obes 2013;6:327-38

13. Centers for Disease Control and Prevention. National Diabetes Statistics Report: Estimates of Diabetes and Its Burden in the United States, 2014. Atlanta, GA: Centers for Disease Control and Prevention, 2014.

14. Portillo-Sanchez P, Bril F, Maximos M, et al. High prevalence of nonalcoholic fatty liver disease in patients with type 2 diabetes mellitus and normal plasma aminotransferase levels. J Clin Endocrinol Metab 2015;100:2231-8. 